\title{
Deterioration of signal detectability during a vigilance task as a function of background event rate'
}

JANE F. MACKWORTH

DEFENCE RESEARCH MEDICAL LABORATORIES, TORONTO, CANADA

\begin{abstract}
In the course of a $30 \mathrm{~min}$. session a significant decrease was found in detection and detectability ( $\left.d^{\prime}\right)$ of a slighter brighter flash against a background flash rate of $200 / \mathrm{min}$. No such decrement occurred when the background flash rate was $40 / \mathrm{min}$. In both cases the signal rate was $6 / \mathrm{min}$ 。
\end{abstract}

\section{Problem}

During a vigilance task the probability of detecting a signal deteriorates as a function of time on task (N. H. Mackworth, 1961). This deterioration may represent a reduction in the ability of the $\mathrm{S}$ to detect the signal, or a reduction in his willingness to give a positive response. According to the Theory of Signal Detection (see Swets, 1964) the detectability of the signal can be measured by the statistic d', which depends upon both the probabilities of a correct $\left(\mathrm{P}_{\mathrm{SN}}(\mathrm{A})\right)$ and of an incor$\operatorname{rect}\left(\mathrm{P}_{\mathrm{N}}(\mathrm{A})\right)$ positive response。

Using the d' measure, Mackworth \& Taylor (1963) and Mackworth (1965) found that the detectability of brief pauses in the continuous steady movement of a clock hand consistently decreased with time during a run. This deterioration was independent of the assumptions made as to the possible number of false alarms over a wide range from $12 / \mathrm{min}$. to $360 / \mathrm{min}$. On the other hand, Broadbent \& Gregory (1963) studied detection of a brighter flash against a slow background flash rate of 43 flashes per min.; they found no changes in probability of detection or of $d^{\prime}$ during the run. They did obtain marked increases in $\beta$, an index of caution, during the run. Similarly, Taylor (1965) has shown that data published by Wiener, Poock, \& Steele (1964) indicated no changes in 'd' during a vigilance task, but a considerable increase in $\beta$. Here again the task required a decision every $1.3 \mathrm{sec}$. It therefore seemed likely that the background or event rate was an important factor in determining deterioration in d' during a run.

The following experiment was designed to study the effect of the background event rate upon the detectability of brief signals at the beginning and end of a $30 \mathrm{~min}$. run.

\section{Method}

The display consisted of a pair of discs of light flashing simultaneously side by side. The discs were produced by neon bulbs placed immediately behind a piece of thin cardboard, whose front face was lit by a 15-w bulb shining from behind $S$. The contrast between the discs of light and their surround was low, so that after-images were reduced. The discs were each $1 / 2$ in in diameter, with a $1 / 2$ in between them. The signal was a brighter flash of the left hand disc. Two flash rates were used, a fast rate of $200 / \mathrm{min}$. and a slow rate of $40 / \mathrm{min}$. The signals were given at a rate of $6 / \mathrm{min}$., with a signal interval varying from $3 \mathrm{sec}$. to $30 \mathrm{sec}$., and a rectangular distribution of intervals.

A preliminary experiment showed that detection of a signal was much easier with the slow background rate, as has been shown by Jerison \& Pickett (1964). Since it was desired to study decrement from approximately the same initial detection level, this effect of background rate was reduced by increasing the duration of the flashes with the fast rate. That is, the duration of the flashes with the fast rate was $0.15 \mathrm{sec}$., while the duration with the slow rate was $0.08 \mathrm{sec}$. The intensities of the flashes were the same in both conditions.

Fourteen housewives were each tested under both conditions, either condition being received in one of two separate sessions. Half the Ss were tested with the fast flash rate first. In each session $\mathrm{S}$ was given $10 \mathrm{~min}$. dark adaptation and then about 5 min. practice with knowledge of results. When $\mathrm{S}$ saw a signal she said 'Now' to E, who sat behind a screen. Responses were recorded as correct if they were made within $1-1 / 2 \mathrm{sec}$. after the signal. With the fast flash rate there were thus 170 flashes/min. to which a response was a false alarm, and with the slow rate $34 / \mathrm{min}$.

Each test run lasted for $30 \mathrm{~min}$. No knowledge of results was given during the test run.

\section{Results}

The data from two Ss were not included in the analysis because their false alarm rates were abnormally high. Data from the remaining 12 Ss are shown in Table 1 in

TABLE I Effect of Flash Rate upon Changes in Detection, False Alarms (FA), and Detectability (d') During Prolonged Observation

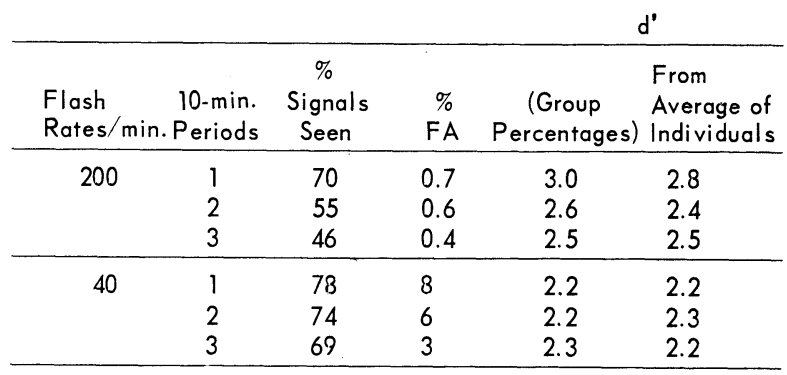


terms of the percentages of correct and incorrect (False Alarms) positive responses. The Wilcoxon Signed-ranks Test (Siegel, 1956, p. 75), was used to compare the differences between the first and last $10 \mathrm{~min}$. of each run.

With the fast flash rate a significant decrease $(p<0.01)$ from first to last $10 \mathrm{~min}$. was found in all three measures, that is, in the percentages of correct and incorrect positive responses, and in the detectability (d') values obtained for 10 individual Ss from these data. The d' values shown in Table 1 are both those obtained from the group percentages, and also averages of individual $d^{\prime}$ values.

With the slow flash rate the only significant change observed during the run was a reduction in the number of false alarms. Since there was no effe tive change in $\mathrm{d}^{\prime}$, this can be interpreted as a change in $\beta$, as found by Broadbent \& Gregory (1963). The actual numbers of false alarms given in the first $10 \mathrm{~min}$. with the slow flash rate were about double those given with the fast flash rate. This difference was, of course, exaggerated when percentages instead of numbers were used. It can be seen that while the percentage detected was higher with the slow flash rate, even though the signal was shorter, the detectability of the signal was lower than with the fast flash rate.

\section{Discussion}

The main finding is that during a $30 \mathrm{~min}$. run there was a significant decrease in both the probability of detection and the detectability of the signal when the event rate was $200 / \mathrm{min}$. When the event rate was $40 / \mathrm{min}$. no significant changes were found in either of these measures. Thus the decline in detectability of a brief signal (less than $0.2 \mathrm{sec}$.) found with the continuous clock has also been found with a light flashing at $200 / \mathrm{min}$. Ss complained of visual difficulties even though contrast effects were as low as possible. Thus the decline observed may be similar to the 'blurring' effect discussed by Jerison et al (1965). A similar quantitative change over time has been found in a variety of tasks (c.f. Mackworth, 1964), and it is possible that it represents an adaptation phenomenon. Jerison et al (1965) have also pointed out that intermittent periods of nonattention ('distraction') lead to reductions in the probabilities of both detections and false alarms; this is probably the main cause of deterioration in detection of signals against a relatively slow background event rate, as found in the classical vigilance task. Such 'distraction' leads to marked changes in $\beta$, with very little change in d'.

It is also possible to interpret the data by the hypothesis that it is the ratio of signal to non-signal events that affects the decrement, just as this ratio affects the initial probability of detection (Colquhoun, 1961, Jerison \& Pickett, 1964). This explanation appears inadequate, however, because Wiener et al (1964) used a signal-to-event ratio of $1 / 80$, and no d' decrement occurred (Taylor, 1965). It therefore seems likely that the decrement in $d^{\prime}$ demonstrated here is related to the background rate rather than to the ratio of signal to non-signal events.

\section{References}

Broadbent, D. B., \& Gregory, M. Vigilance considered as a statistical decision. Brit. J. Psychol., 1963, 54, 309-323.

Colquhoun, W. P. The effect of 'unwanted' signals on performance in a vigilance task. Ergonomics, 1961, 4, 41-51.

Jerison, H. J., \& Pickett, R. M. Vigilance: the importance of the elicited observing rate. Science, 1964, 143, 970-971.

Jerison, H. J., Pickett, R. M., \& Stenson, H. H. The elicited observing rate and decision processes in vigilance. Hum. Factors, 1965 , in press.

Mackworth, J. F. Performance decrement in vigilance, threshold and high-speed perceptual motor tasks. Canad. J. Psychol., $1964,18,209-223$.

Mackworth, J. F. Decision interval and signal detectability in a vigilance task. Canad. J. Psychol., 1965, 19, 111-117.

Mackworth, J. F., \& Taylor, M. M. The d'measure of signal detectability in vigilance-like situations. Canad. J. Psychol., 1963, $17,302-325$

Mackworth, N. H. Researches on the measurement of human performance. In H. W. Sinaiko (Ed.), Selected papers on human factors in the design and use of control systems. New York: Dover Publications, 1961.

Siegel, S. Nonparametric statistics for the behavioral sciences. New York: McGaw-Hill, 1956.

Swets, J. A. (Ed.) Signal detection and recognition by human observers. New York: Wiley, 1964.

Taylor, M. M. Detectability measures in vigilance: comment on a paper by Wiener, Poock, and Steel. Percept. mot. Skills, 1965, 20, 1217-1221.

Wiener, E. L., Poock, G. K., \& Steele, M. Effect of time-sharing on monitoring performance; simple mental arithmetic as a loading task. Percept. mot. Skills, 1964, 19, 435-440.

Note

1. Defence Research Medical Laboratories Research Paper No. 616 PCC No. D77-94-20-42. 